\title{
Disassociare, dissociare
}

\section{Domenico Proietti}

PUBBLICATO: 20 SETTEMBRE 2019

\section{Quesito:}

I diversi quesiti pervenuti sulla coppia verbale disassociare/dissociare vertono sul rapporto semantico tra i due verbi (percepiti come sinonimi da alcuni) e sulla loro frequenza d'uso (da parte di altri si rileva la minor diffusione del verbo disassociare e quindi si chiede se sia "più giusto" o "meglio" usare dissociare, in quanto forma della "lingua corrente"). Inoltre, si osserva che nella forma disassociare, avvertita come inusuale, la sostituzione del "prefisso ad-con il prefisso dis-" crea "uno strano cumulo di prefissi contraddittori".

\section{Disassociare, dissociare}

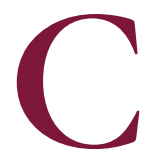

onviene partire dalla storia dei due verbi. Il più antico è dissociare, continuatore diretto del latino dissociāre, di forma attiva e usato sia in senso concreto (dissociare intus partes 'separare le parti intimamente connesse tra loro', Lucrezio, De rerum natura, III 8Io), sia in senso estensivo-figurato ([morum $]$ dissimilitudo dissociat amicitias 'la differenza dei costumi disgiunge le amicizie', Cicerone, De amicitia, XX 74 ).

In italiano, la forma attiva e il senso concreto del verbo ('dividere, staccare, separare') sono sporadicamente attestati dal XIV secolo; al Seicento risalgono accezioni specifiche nel linguaggio scientifico (in particolare nella chimica, con il significato ancora in uso di 'scindere un composto nei suoi costituenti', cfr. GDLI, vol. IV, p. 776). Più recenti (a partire dal Settecento) sono gli usi, estensivi, nella forma media o riflessivo-reciproca dissociarsi ("il popolo [...] si dissociò, e divise in vari partiti", Carlo Botta, I797), anche con l'indicazione della persona e della cosa da cui ci si dissocia ("egli [il Signore] ricomincia in voi l'opera della vostra salute, e voi vi dissociate da lui dopo i primi passi della vostra conversione, e ritornate al peccato", Esame pratico ed istruttivo sui peccati che si commettono, I809). All'Ottocento risale il derivato dissociazione, già presente nella prosa di Giuseppe Mazzini ("cesserà quello stato di dissociazione e di diffidenza che ci affatica", Fede e avvenire, I835), largamente usato in chimica e fisica (dissociazione elettrolitica, termica, ecc.), passato dalla filosofia alla psicologia (per es. nella locuzione dissociazione psichica, derivata dalle teorie di Sigmund Freud) e infine esteso (a partire dal D.L. 5 dicembre I979, n. 625) a indicare la condizione giuridica dei terroristi (dissociati) che, ripudiando il metodo e la strategia della lotta armata e rendendo piena confessione dei reati commessi, si adoperano nel corso del processo per eliminare o attenuare le conseguenze dei reati da loro commessi, senza fornire (a differenza dei "collaboratori di giustizia o "pentiti") indicazioni o informazioni su responsabilità di terzi.

Per il verbo disassociare, d'uso assai meno frequente di dissociare, non si risale, invece, oltre la metà dell'Ottocento (se ne trova un esempio in forma sostantivata in un discorso di Cavour al Senato Subalpino, il 5 marzo ı 854: "è bene il disassociare l'idea delle lotterie dall'idea di beneficenza"). Nel Dizionario della lingua italiana di Nicolò Tommaseo e Bernardo Bellini (vol. II, I865, p. 236) si ha una precoce registrazione lessicografica del verbo, con una descrizione ancora essenzialmente valida. Rilevandone l'uso "segnatam[ente, cioè prevalentemente] rifl[essivo]", il verbo viene così spiegato: "Disdire l'associazione, Non voler più essere associato alla compra d'un libro, alla lettura di giornale, o 
altra spesa. Altro dunque da Dissociare"; e non senza ironia si osserva anche: "Taluni, per la più spiccia, si disassociano tacitamente col non pagare. Montaigne l'ha in altro senso".

Pienamente fondata, comunque, dal punto di vista semantico ed etimologico, è la distinzione di disassociare da dissociare: quest'ultimo, continuatore diretto di un verbo latino, d'antica attestazione in italiano e con una più ampia gamma d'usi e accezioni. Con ogni probabilità disassociare è un prestito dal francese désassocier, attestato dal XVI sec. (come si può dedurre, tra l'altro anche dal riferimento nel Tommaseo-Bellini a un famoso passo del terzo libro degli Essais di Montaigne): data questa particolare trafila, non si può quindi parlare, in riferimento a disassociare, di "strano cumulo di prefissi contraddittori" come scrive una nostra lettrice.

\section{Cita come:}

Domenico Proietti, Disassociare, dissociare , "Italiano digitale", 2019, X, 2019/3 (luglio-settembre) DOI: $10.35948 / 2532-9006 / 2020.3206$ 